\title{
Antagonistic Regulation of Arabidopsis Growth by Brassinosteroids and Abiotic Stresses
}

\author{
Yuhee Chung ${ }^{1,4}$, Soon II Kwon ${ }^{2}$, and Sunghwa Choe ${ }^{1,2,3, *}$
}

To withstand ever-changing environmental stresses, plants are equipped with phytohormone-mediated stress resistance mechanisms. Salt stress triggers abscisic acid (ABA) signaling, which enhances stress tolerance at the expense of growth. ABA is thought to inhibit the action of growth-promoting hormones, including brassinosteroids (BRs). However, the regulatory mechanisms that coordinate $A B A$ and $B R$ activity remain to be discovered. We noticed that $A B A$-treated seedlings exhibited small, round leaves and short roots, a phenotype that is characteristic of the BR signaling mutant, brassinosteroid insensitive1-9 (bri1-9). To identify genes that are antagonistically regulated by $A B A$ and BRs, we examined published Arabidopsis microarray data sets. Of the list of genes identified, those upregulated by ABA but downregulated by BRs were enriched with a BRRE motif in their promoter sequences. After validating the microarray data using quantitative RTPCR, we focused on RD26, which is induced by salt stress. Histochemical analysis of transgenic Arabidopsis plants expressing RD26pro:GUS revealed that the induction of GUS expression after $\mathrm{NaCl}$ treatment was suppressed by co-treatment with BRs, but enhanced by co-treatment with propiconazole, a BR biosynthetic inhibitor. Similarly, treatment with bikinin, an inhibitor of BIN2 kinase, not only inhibited RD26 expression, but also reduced the survival rate of the plant following exposure to salt stress. Our results suggest that $A B A$ and BRs act antagonistically on their target genes at or after the BIN2 step in BR signaling pathways, and suggest a mechanism by which plants finetune their growth, particularly when stress responses and growth compete for resources.

\footnotetext{
${ }^{1}$ School of Biological Sciences, College of Natural Sciences, Seoul National University, Seoul 151-747, Korea, ${ }^{2}$ Convergence Research Center for Functional Plant Products, Advanced Institutes of Convergence Technology, Suwon 443-270, Korea, ${ }^{3}$ Plant Genomics and Breeding Institute, Seoul National University, Seoul 151-921, Korea, ${ }^{4}$ Present address: Department of Biology, School of Arts and Sciences, University of Pennsylvania, Philadelphia, USA

*Correspondence: shchoe@snu.ac.kr

Received 15 May, 2014; revised 6 September, 2014; accepted 11 September, 2014; published online 5 November, 2014
}

Keywords: ABA, abiotic stress, BIN2, brassinosteroids, RD26, Root

\section{INTRODUCTION}

Due to their sessile nature, plants have developed strategies to cope with abiotic challenges and biotic stresses (Chung et al., 2012; Kim et al., 2014; Maharjan and Choe, 2011). Plants exposed to abiotic stresses display severe growth retardation and reduced productivity. Growth is regulated by plant hormones, which modify endogenous programs in response to exogenous signals. However, the hormone-dependent mechanisms by which growth is inhibited under stress conditions are not fully understood. The molecular mechanisms that impart tolerance to water stress can be divided into abscisic acid (ABA)dependent and ABA-independent pathways (Shinozaki and Yamaguchi-Shinozaki, 2007). ABA plays vital roles in adaptation to environmental changes, seed dormancy, and the regulation of stomatal closure (Grill and Himmelbach, 1998; Lee and Luan, 2012).

Under stressed conditions, plants rapidly produce $A B A$, which stimulates the resistance mechanism. In the ABAdependent pathway, ABA binds to soluble receptors of the PYRABACTIN RESISTANCE1 (PYR1)/PYR1-LIKE (PYL)/REGULATORY COMPONENTS OF ABA RECEPTORS (RCARs) family, which induces the de novo synthesis of ABA through 9cis epoxycarotenoid dioxygenase (NCED) as part of a positive feedback mechanism (Ma et al., 2009; Park et al., 2009). Once ABA activates SnRK2s, SnRK2s phosphorylate and thereby activate the transcription of ABA-dependent transcription factors, such as ABA-responsive element binding factors (ABFs/AREBs) (Furihata et al., 2006; Uno et al., 2000). ABFs/AREBs then bind to the $A B A$ response element (ABRE) in the promoter region of their target genes (Mundy et al., 1990). These conserved elements contain PyACGTGGC (Busk and Pages, 1997; Guiltinan et al., 1990; Yamaguchi-Shinozaki et al., 1990) and ACGTG[G/T]C (Hobo et al., 1999) residues. ABA regulates approximately $10 \%$ of the genes in Arabidopsis, and many of these genes are also regulated by other hormones (Nemhauser et al., 2006).

In addition to ABFs/AREBs, other ABA-induced transcription factors are known to participate in the ABA response and stress tolerance. For instance, the NAC (NAM, ATAF1/2 and CUC2) transcription factor family, which largely consists of NO APICAL MERISTEM (NAM) and Arabidopsis thaliana transcription activation factor (ATAF1/2) transcription factors, is known to function in the stress response (Aida et al., 1997). ATAF1/ANAC002 directly regulates $A B A$ biosynthesis through the transcriptional activation of NCED3 (Jensen et al., 2013; Tran et al., 2004). 
$R D 26$ is a member of the ATAF family and is reported to function in ABA-dependent stress-response pathways (Fujita et al., 2004; Ooka et al., 2003).

Brassinosteroids (BRs) are a class of plant steroidal hormones (Chung and Choe, 2013). Like mammalian steroid hormones such as estrogen, ecdysone, and progesterone, BRs play key roles in plant development, regulating processes such as cell elongation, vascular system differentiation, senescence, and stress responses (Choe, 2006; Clouse and Sasse, 1998). BRs and other phytohormones have numerous target genes in common, and complex crosstalk mechanisms exist among these hormone signal transduction pathways (Chung et al., 2011; Nemhauser et al., 2006). Brassinolide (BL), the most active form of BRs, binds to an island domain in the extracellular domain of BRASSINOSTEROID INSENSITIVE 1 (BRI1) (Kinoshita et al., 2005; Sun et al., 2013). In the absence of BL, $\mathrm{BKI} 1$ binds to the cytoplasmic kinase domain of BRI1 and thereby inhibits its phosphorylation function (Wang et al., 2008). However, when BL binds to BRI1, BKI1 is released from BRI1 and BRI1 forms a heterodimer with BRI1-ASSOCIATED-KINASE 1 (BAK1) (Russinova et al., 2004; Wang and Chory, 2006). Then, BR-SIGNALING KINASE 1 (BSK1) is released from the cytoplasmic domain of BRI1 and transduces the signal into the nucleus via a protein phosphatase, bri1 SUPPRESSOR1 (BSU1) (Kim et al., 2009; Tang et al., 2008). BSU1 inactivates the BRINSENSITIVE 2 (BIN2) kinase that acts as a negative regulator in BR signaling pathways (Choe et al., 2002; Li and Nam, 2002; $\mathrm{Li}$ et al., 2001). BIN2 negatively regulates BR-specific transcription factors, including BZR1 and BES1 by phosphorylation (Kim et al., 2009), and is degraded in response to BL (Peng et al., 2008). BZR1 binds to the BR response element (BRRE) motif (CGTG[T/C][G/A]) (He et al., 2005) to both activate BR response genes and inhibit BR biosynthetic genes, including DWF4 (Choe et al., 2001; Kim et al., 2006; 2013). Weak alleles of bri1 include bri1-5 (Noguchi et al., 1999) and bri1-9, and exhibits a semi-dwarf phenotype (Jin et al., 2007).

In contrast to the ABA pathway, the majority of signaling components have been elucidated in the BR signaling cascade (Vriet et al., 2013). Given the importance of ABA and BRs, much research has focused on identifying the mechanism of crosstalk between BRs and ABA. It has been demonstrated that $A B A$ and $B R s$ antagonistically regulate each other during seed germination and root growth inhibition (Steber and McCourt, 2001; Zhang et al., 2009)

In this study, we sought to understand the mechanisms by which $B R$ s and $A B A$ interact. First, we selected putative marker genes that are antagonistically regulated by BRs and ABA from a microarray database deposited in AtGenExpress (Nemhauser et al., 2006). Secondly, we found that chemical inhibition of BIN2 decreased the plant's tolerance to salt stress, suggesting that BIN2 is involved in ABA-mediated salt tolerance processes. Based on our findings, we propose a model in which transcription factors that bind to common target genes and are specific to either BRs or ABA are antagonistically regulated by each other to bring about ABA-dependent stress responses.

\section{MATERIALS AND METHODS}

\section{Plant material and growth conditions}

Arabidopsis plants of Columbia ecotype were grown in $0.5 \times$ Murashige and Skoog (MS) medium containing $0.5 \%$ sucrose and $0.8 \%$ plant agar. Plants or seedlings were kept in a growth room at $22^{\circ} \mathrm{C}$ with a $16 \mathrm{~h}$ light/ $8 \mathrm{~h}$ dark cycle. To measure the survival rate in salt media, seeds were germinated and grown in MS agar medium and transferred to medium containing the indicated concentrations of hormone or chemical. After 1 day, seedlings were transferred again to medium containing $\mathrm{NaCl}$. epi-Brassinolide $(\mathrm{BL})$ was dissolved in $\mathrm{DMSO}$, and $\mathrm{ABA}$ stock was prepared in ethyl alcohol (EtOH). The controls contained the same volume of DMSO or EtOH as chemicals added to the treated aliquots, and considered a mock treatment.

\section{Quantitative RT-PCR}

RNA extraction and cDNA synthesis were conducted according to a previous report (Chung et al., 2012). Quantitative RT-PCR analysis was performed using SYBR-mix (KAPA Biosystems). UBQ10 was used as an internal control. Primer sets used for PCR are listed in Supplementary Table S2.

GUS histochemical assay and quantification of GUS activity Five-day-old RD26pro:GUS transgenic seedlings were first treated with the indicated concentrations of hormone or chemical. Following pre-incubation, each seedling was transferred to $\mathrm{NaCl}$-containing agar medium supplemented with the same concentration of hormone or chemical. After 3 days, seedlings were transferred to GUS buffer (1 mM 5-bromo-4-chloro-3indoyl- $\beta-d-G l c U A, 100 \mathrm{mM}$ sodium phosphate $(\mathrm{pH} 7), 5 \mathrm{mM}$ potassium ferrocyanide, $5 \mathrm{mM}$ potassium ferricyanide, $10 \mathrm{mM}$ EDTA, and $0.1 \%(\mathrm{v} / \mathrm{v})$ Triton $\mathrm{X}-100)$ and incubated for $2 \mathrm{~h}$. Serially-diluted $\mathrm{EtOH}$ was used to clear the chloroplasts and to reduce background staining. Micrographs were taken using a stereomicroscope (Olympus). To quantify the in vivo GUS activity of RD26pro:GUS in each treatment, $0.5 \mathrm{~cm}$ of the root tip of treated seedlings was excised and transferred to 96-well plates pre-filled with a substrate solution (Blazquez et al., 1998). Seedlings in the substrate solution were incubated for $1 \mathrm{~h}$ at $37^{\circ} \mathrm{C}$ and the reaction was stopped by the addition of $100 \mu \mathrm{l}$ of cold $0.2 \mathrm{M} \mathrm{Na}_{2} \mathrm{CO}_{3}$ solution. The fluorescence intensity was measured using a fluorescence spectrophotometer (Varian, USA) with an excitation wavelength of $360 \mathrm{~nm}$ and an emission wavelength of $465 \mathrm{~nm}$. The values of 12 seedlings were averaged and plotted with their standard error. The standard curve was calculated using known concentrations of 4methylumbelliferol solution.

\section{Motif prediction by MEME}

MEME (http://meme.sdsc.edu) was used to search for motifs conserved in the promoter region $1000 \mathrm{bp}$ upstream of the start codons of genes antagonistically regulated by $A B A$ and $B R$ (Bailey and Elkan, 1995). A MEME search revealed that the optimum width for motifs was 6 to $9 \mathrm{bp}$.

\section{RESULTS}

Morphological similarity between ABA-treated seedlings and the BR-deficient dwarf mutant

We observed that treatment of Arabidopsis seedlings with ABA often resulted in phenotypes that resembled those of BRdeficient dwarf mutants. The small, curled leaves of ABAtreated Col wild-type plants were similar to those of mocktreated bri1-9 plants (Figs. 1A and 1B), which bear a loss-offunction mutation in the BR receptor (Jin et al., 2007). Previously, we also showed that $B R$ mutants are more sensitive to ABA treatment (Choe et al., 2002). These findings suggest that ABA and $\mathrm{BRs}$ interact to regulate Arabidopsis growth.

Because ABA treatment mimicked the BR mutant phenotype, we reasoned that bri1-9 would be more sensitive to $A B A$ than the wild type. To test this, we performed a dose-response assay in which we examined the key characteristics of seedling development, including root growth, germination, and post- 
A

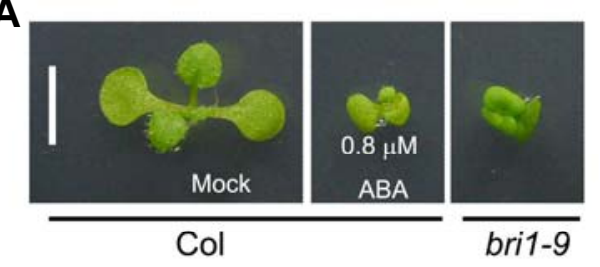

D

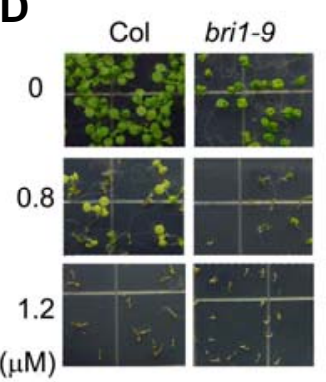

ABA
B

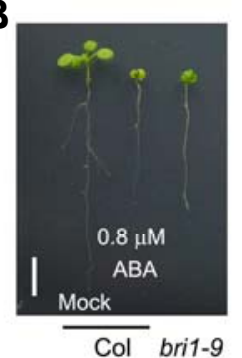

E

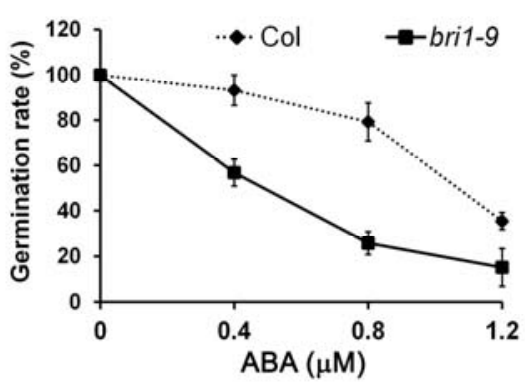

C
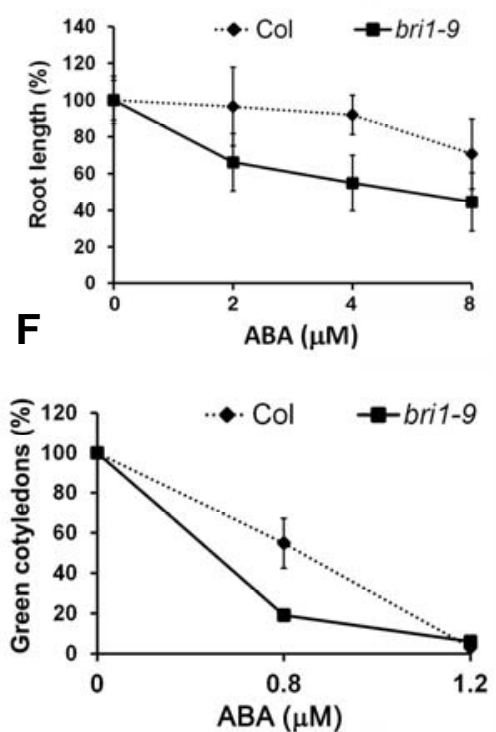

Fig. 1. Response of wild-type and bri1-9 seedlings to $A B A$ treatment. (A) Top view of Arabidopsis wild-type seedlings treated with mock or $A B A$, and bri1-9 treated with mock only. Scale bar $=0.5 \mathrm{~cm}$. (B) Whole seedling morphology of 10-day-old Col and bri1-9 plants. From left, mocktreated wild type, ABA-treated wild type, and bri1-9. Scale bar $=1 \mathrm{~cm}$. (C) Statistical representation of the dose-dependent effect of ABA on root growth. Seedlings were germinated on MS medium before being transferred to medium containing $0,2,4$, or $8 \mu \mathrm{M} A B A$ for a further 5 days of growth. The growth of bri1-9 roots was retarded to a greater extent by ABA treatment than was that of wild-type roots. Values show root length relative to that of an untreated wild-type control. (D) Germination of Col and bri1-9 on ABA-supplemented media. Photographs were taken ten days after sowing. Germination is suppressed to a greater extent in the bri1-9 plates. (E) Statistical analysis of germination rates in response to different ABA doses. Seeds with visible radicles were scored as successful germination events. Average values are represented with standard deviations of three independent biological replicates. Again, bri1-9 was more sensitive than the wild type to ABA treatment. (F) Establishment of green cotyledons in the presence of ABA. Seedlings with green cotyledons were scored. Error bars in (C, E, and F) are standard deviations $(n=3)$.

germination growth, under various concentrations of ABA. The root growth rate of wild-type plants was reduced in response to ABA treatment in a dose-dependent manner, and the degree of reduction was much greater in bri1-9 (Fig. 1C). In addition, we scored the percentage of seeds with emerged radicles 3 days after sowing on mock, 0.4, 0.8, and $1.2 \mu \mathrm{M}$ ABA medium (Fig. 1D). On $0.8 \mu \mathrm{M} \mathrm{ABA}, 80 \%$ of the Col seeds germinated, whereas only $25 \%$ of bri1- 9 seeds did, suggesting that bri1-9 is more sensitive to exogenous ABA treatment than is the wild type (Fig. 1E). Of the germinated seeds, only $55 \%$ and $19 \%$ of the cotyledons of wild-type and bri1-9 seedlings remained green, respectively, at $0.8 \mu \mathrm{M}$ ABA (Fig. 1F). Thus, the growth of bri1-9 plants was more sensitive to ABA than the wild type, suggesting that $A B A$ controls $B R$ signaling pathways to achieve optimal growth and development during the ABA-mediated stress response.

\section{BRs and ABA antagonistically regulate overlapping sets of} genes

To identify the genes that are oppositely regulated by ABA and BRs, we analyzed a set of publicly available microarray data that were reported by Nemhauser et al. (2006). They reported that 383 genes upregulated by BRs, 268 were downregulated by BRs, 1440 were upregulated by $A B A$, and 1476 were downregulated by ABA (Nemhauser et al., 2006). Because BRs and ABA have a tendency to reverse the effects on Arabidopsis growth (Fig. 1), we focused on genes that respond oppositely to BRs and ABA. We determined the union (62) of genes downregulated by $B R$ s and upregulated by $A B A$, and the union (50) upregulated by BRs and downregulated by ABA (Fig. 2A). These genes were chosen for further investigation.

Because BZR1 can function either as an inducer or repressor of BR-responsive genes ( $\mathrm{He}$ et al., 2005), we determined the number of BR-response elements (BRREs, CGTG[TC][GA], (He et al., 2005)) in the promoter DNA sequences of these genes (Fig. 2B). Whereas $25 \%$ of genes in the whole genome contained the BRRE motif, $48 \%$ of genes upregulated by ABA and downregulated by BR contained this motif (Fig. 2B). Since the BRRE was not enriched in the group of genes downregulated by $A B A$ and upregulated by $B R$, we propose that genes that are upregulated by $A B A$ and downregulated by $B R$ are likely targeted by BZR1 through direct binding to BRRE in the promoter sequences.

We next determined the frequency of the BRRE in genes reported to be regulated by other hormones or a biosynthetic precursor of hormone, such as 1-aminocyclopropane-1carboxylic acid (ACC), indole-3-acetic acid (IAA), methyl jasmonate (MeJA), gibberellins (GA), and cytokinins (CK). Whereas the percentage of genes possessing the BRRE is enriched in the ABA-upregulated group $(36.4 \%)$ and BRdownregulated group $(35.2 \%)$ compared to the whole genome control $(25 \%)$, the percentage was similar for other hormoneresponse genes (Table 1), except for those upregulated by MeJA, suggesting that BRs negatively regulate JA signaling, as previously noted (Kim et al., 2013).

Of the genes upregulated by ABA and downregulated by BRs, 
Antagonistic Interaction between BRs and ABA

Yuhee Chung et al.

Table 1. Proportion of genes containing the BRRE

\begin{tabular}{|c|c|c|c|c|c|c|c|c|c|c|c|c|c|c|}
\hline \multirow[b]{2}{*}{ Up or Down } & \multicolumn{2}{|c|}{$\mathrm{ABA}$} & \multicolumn{2}{|c|}{ BRs } & \multicolumn{2}{|c|}{$\mathrm{ACC}$} & \multicolumn{2}{|c|}{ IAA } & \multicolumn{2}{|c|}{ GA } & \multicolumn{2}{|c|}{ MeJa } & \multicolumn{2}{|c|}{ CK } \\
\hline & $\mathrm{U}$ & $\mathrm{D}$ & $\mathrm{U}$ & $\mathrm{D}$ & $\mathrm{U}$ & $\mathrm{D}$ & $\mathrm{U}$ & $\mathrm{D}$ & $\mathrm{U}$ & $\mathrm{D}$ & $\mathrm{U}$ & $\mathrm{D}$ & $\mathrm{U}$ & $\mathrm{D}$ \\
\hline No. genes & 1440 & 1476 & 268 & 383 & 167 & 365 & 430 & 355 & 40 & 82 & 806 & 701 & 332 & 163 \\
\hline $\begin{array}{l}\text { BRRE-containing } \\
\text { gene }\end{array}$ & 524 & 356 & 59 & 135 & 45 & 87 & 123 & 78 & 11 & 26 & 272 & 181 & 81 & 46 \\
\hline BRRE/Total & 36.4 & 24.1 & 22.0 & 35.2 & 26.9 & 23.8 & 28.6 & 22.0 & 27.5 & 31.7 & 33.7 & 25.8 & 24.4 & 28.2 \\
\hline
\end{tabular}

A
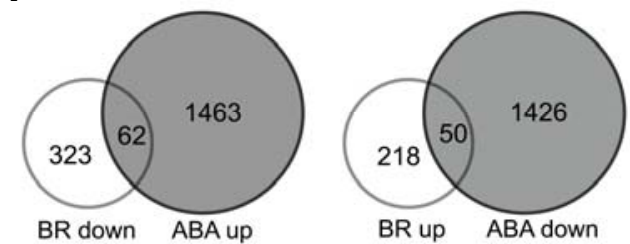

C

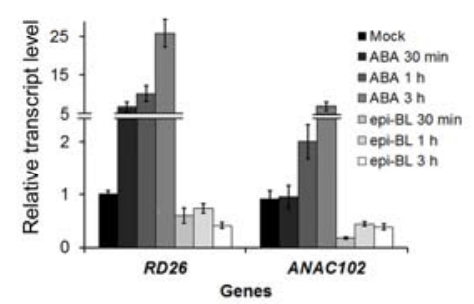

D

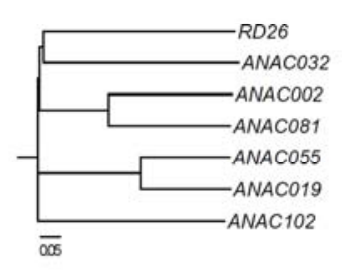

B

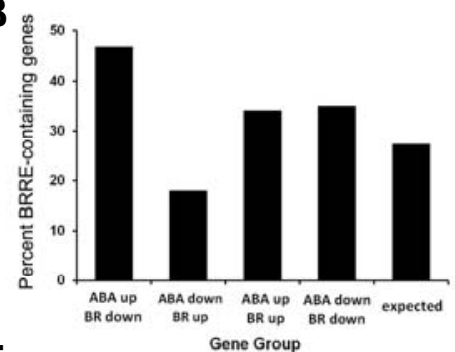

E

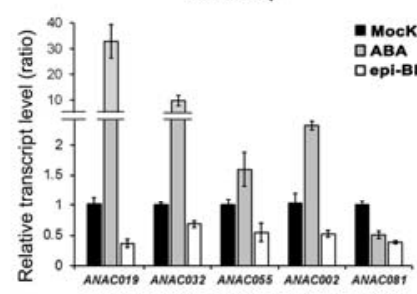

Fig. 2. Identification of genes co-regulated by $B R s$ and $A B A$ in publicly available microarray data. (A) Venn diagram of ABA- and BRresponsive genes. Genes that are antagonistically regulated by these hormones were selected from previously-reported data sets (Nemhauser et al., 2006). Left, Venn diagram of genes downregulated by BRs but upregulated by ABA; right, genes upregulated by BRs but downregulated by ABA. Fisher's exact test was conducted to determine the $p$-value of overlapping genes $(P<0.09$ for genes upregulated by $A B A$ and downregulated by BR; $P<0.0051$ for genes downregulated by $A B A$ and upregulated by $B R$ ). (B) Enrichment of the BR-response element (BRRE) in the -1000 bp promoter sequences of 62 and 50 genes co-regulated by BRs and ABA, respectively. The percentage of genes that harbor at least one BRRE (CGTG[T/C][G/A]) was determined by searching the Arabidopsis genome sequence database. (C) Timecourse analysis of RD26 and ANAC102 transcript levels in seven-day-old wild-type seedlings treated with $1 \mu \mathrm{M}$ epi-BL or $50 \mu \mathrm{M}$ ABA in liquid MS media. Results were normalized using UBQ10, and converted to values relative to a mock-treated control. Data are mean \pm standard deviation of three technical replicates. (D) RD26 belongs to the NAC transcription factor family, which is composed of at least 94 members (Christianson et al., 2010). Of the entire phylogenetic tree of this gene family, a clade containing RD26 is shown. The length of the bar indicates the number of changes in amino acid residues per unit length of the horizontal branches. (E) qRT-PCR analysis of the expression of ANAC genes clustered with RD26 in (D). UBQ10 levels were used as loading controls. Besides ANAC081, all tested ANAC genes responded similarly to $R D 26$; i.e., they were upregulated in response to $A B A$, and downregulated after exposure to epi-BL.

seven encoded $A B F$ transcription factors (Supplementary Table S1). qRT-PCR analysis showed that RD26 and ANAC102 transcription was induced and repressed by $A B A$ and epi-BL, respectively (Fig. 2C). RD26, ANAC102, and ANAC092 encode $N A C$ transcription factors, which contain a NAC domain (Fig. 2D). Moreover, RD26 and ANAC102 are closely clustered in the phylogenetic tree, belonging to the ATAF subfamily of NAC domain proteins (Fujita et al., 2004). We extended our analysis to other genes in the ATAF subfamily, and found that these genes are also regulated by BRs (Fig. 2E). We measured the transcript level of each gene in response to a 3-h treatment with $\mathrm{ABA}$ or BRs, and found that all the genes except for ANAC081 were downregulated by BRs (Fig. 2E). Although ANAC081 did not respond to epi-BL, it was reported to be upregulated by $\mathrm{NaCl}$ and drought stress (Fujita et al., 2004). Previously, chromatin-immunoprecipitation microarray (ChIP-Chip) experiments revealed that ANAC102, ANAC032, ANAC019, ANAC081, $A N A C 055$, and RD26 are the direct targets of BZR1 (Sun et al., 2010), suggesting that BZR1 directly regulates the expression of these genes. Considering that this subfamily of NACs is regulated by various environmental stimuli in addition to $A B A$, it is likely that BRs regulate this group of genes to balance growth and stress pathways.

Overexpression of RD26 results in a weak BR-deficient mutant phenotype

Similar to BR mutants, 35Spro:RD26 overexpression lines were previously reported to have round leaf blades and short petioles (Fujita et al., 2004). We thus were given the DNA of 35Spro:RD26 and generated the transgenic lines to re-examine the responses after hormone and stress treatments. We confirmed that RD26 overexpression lines exhibited a semi-dwarf 

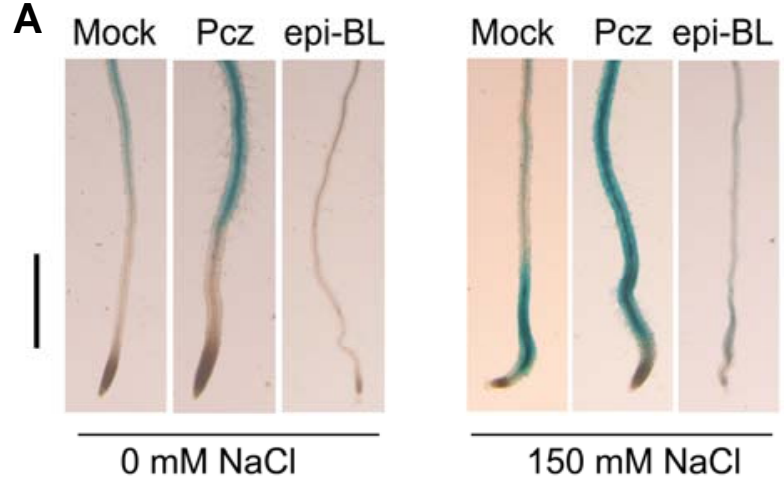

B

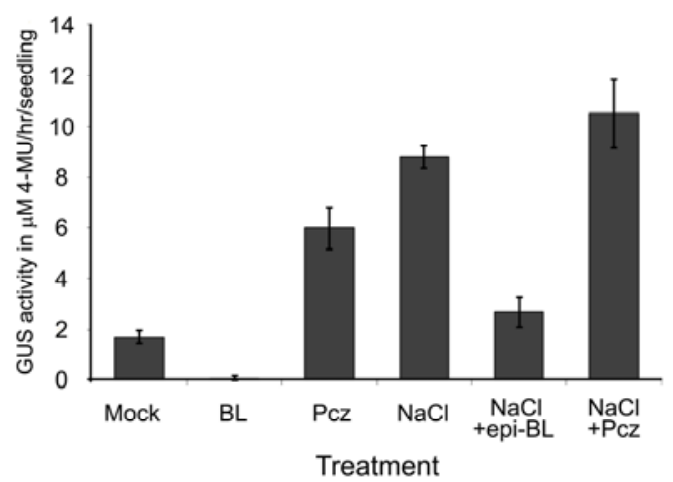

Fig. 3. BR-mediated regulation of RD26. (A) GUS histochemical staining pattern of RD26pro:GUS seedlings. Four-day-old RD26pro:GUS seedlings were transferred to media containing epi$\mathrm{BL}$ or Pcz and further incubated for two days (left panel), or transferred to media containing $150 \mathrm{mM} \mathrm{NaCl}$ plus the indicated chemicals and incubated for two more days. Scale bar $=0.5 \mathrm{~mm}$. (B) Quantitative assay of GUS activity using the bottom $5 \mathrm{~mm}$ of tissue from root tips. Error bars represent $\pm S E(N>12)$.

phenotype, with a reduction in leaf size and inflorescence height (Supplementary Fig. S1A). The phenotype was more pronounced in lines with high levels of 35Spro:RD26 expression, suggesting that these phenotypes are attributable to RD26 (Supplementary Figs. S1B and S1C). A contrasting phenotype was observed when RD26 activity was suppressed by an inhibitory SRDX epitope fused to the carboxy-terminus of RD26. Such plants had elongated petioles and downwardcurled leaves, which are characteristics of constitutive BR mutants (Chung et al., 2012; Fujita et al., 2004; Kim et al., 2013). These findings suggest that the genetic pathways controlling the stress response and growth are tightly coupled by the actions of BRs and ABA.

Interestingly, previous reports showed that overexpression of other transcription factors involved in the response to abiotic stresses, including $A B F 3$ and $A B F 4$, also resulted in a retarded growth phenotype (Kang et al., 2002). Furthermore, overexpression of the $C B F 3$ transcription factor, which is essential for cold stress tolerance, resulted in a semi-dwarf phenotype (Sakuma et al., 2006). Taken together, it is likely that stress tolerance mechanisms, which protect the plant from abiotic stresses, limit energy-requiring BR-dependent growth.
Transcriptional induction of RD26 in salt stress conditions is inhibited by BRs and stimulated by propiconazole treatment

It was reported that $B R$ mutants are hypersensitive to $A B A$. Moreover, BR-overproducing and constitutive BR signaling mutants tend to be less tolerant to stresses (Chung et al., 2012; Kim et al., 2013), suggesting that BR negatively regulates stress responses. Since RD26 responds not only to ABA but also to ABA-dependent water stresses (Fujita et al., 2004), we chose $R D 26$ as a marker gene that is antagonistically regulated by $B R$ and $A B A$ during $A B A$-dependent stresses. To investigate if $R D 26$ transcription is inhibited by BRs when the plant is subjected to stress, we examined $R D 26$ expression in a transgenic Arabidopsis plant expressing RD26pro:GUS. In general, GUS staining was confined to the roots. In mock conditions, the 6day-old seedlings displayed weak GUS activity in the root region above the elongation zone (Fig. 3A). However, when seedlings were treated with the BR biosynthetic inhibitor propiconazole (PCz) (Hartwig et al., 2012) for 3 days, GUS expression became markedly stronger. In contrast, GUS expression was barely detectable in the slender roots of seedlings treated with epi-BL (Fig. 3A).

Previously, it was reported that RD26pro:GUS expression was rapidly induced in all tissues by salt stresses (Fujita et al., 2004). When 6-day-old RD26pro:GUS seedlings were incubated in $150 \mathrm{mM} \mathrm{NaCl}$ for 2 days, GUS staining was strongly detected, especially in the root tip region (Fig. 3A). To determine the effects of Pcz and BL, seedlings were pre-treated with Pcz or $\mathrm{BL}$ for 2 days before being transferred to $\mathrm{NaCl}$ media supplemented with Pcz and epi-BL, respectively. Pretreatment with Pcz synergistically induced the expression of RD26pro:GUS in transgenic seedlings subjected to $150 \mathrm{mM} \mathrm{NaCl}$, indicating that $\mathrm{Pcz}$ accelerates the induction of RD26 expression in response to stress. However, epi-BL pre-treated samples displayed little staining in the roots, with only marginal induction by $\mathrm{NaCl}$.

To quantify the expression of RD26pro:GUS, root tips ( 5 $\mathrm{mm})$ were excised and GUS activity was measured. Consistently, GUS activity was noticeably increased in samples treated with Pcz, whereas almost no signal was detected in epi-BLtreated samples (Fig. 3B). Moreover, treatment with $200 \mathrm{mM}$ $\mathrm{NaCl}$ caused physical damage to plants than treatment with $150 \mathrm{mM} \mathrm{NaCl}$, as tissues disintegrated when the osmotic balance was disrupted. However, the phenotype of roots exposed to $200 \mathrm{mM} \mathrm{NaCl}$ alone for 2 days was different from that of samples pre-treated with epi-BL or Pcz and then exposed to $200 \mathrm{mM} \mathrm{NaCl}$ for 2 days (Supplementary Fig. S2). The roots of Pcz-treated seedlings were harder and thicker than those treated with epi-BL, which were weak and fragile. Taken together, BRs repress RD26 expression, both in ambient and stress conditions, while Pcz enhances RD26 expression.

\section{BIN2 increases salt stress tolerance}

We have demonstrated that $B R s$ repress the expression of the ABA-responsive genes to ameliorate ABA-dependent stress tolerance. To test which component in the BR signaling pathway is the focal point of ABA and BR crosstalk in the salt stress tolerance mechanism, we first examined the salt stress tolerance of wild-type seedlings treated with the BIN2 inhibitors, Lithium (Klein and Melton, 1996; Stambolic et al., 1996) and bikinin (De Rybel et al., 2009). Lithium inhibits BIN2 activity by competing with $\mathrm{Mg}^{2+}$ (Klein and Melton, 1996; Stambolic et al., 1996). In presence of Lithium ( $\mathrm{LiCl}$ ), the effects of $\mathrm{NaCl}$ was greatly enhanced such that seedlings turned yellow and died (Fig. 4A). More quantitatively, the survival rate of seedlings treated with $3 \mathrm{mM} \mathrm{LiCl}$ was $42 \%$ that of control seedlings treat- 
A

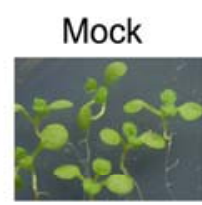

$\mathrm{KCl}$

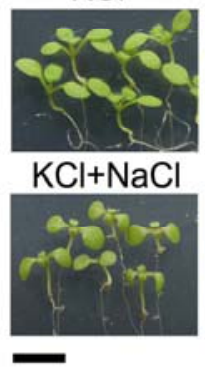

B
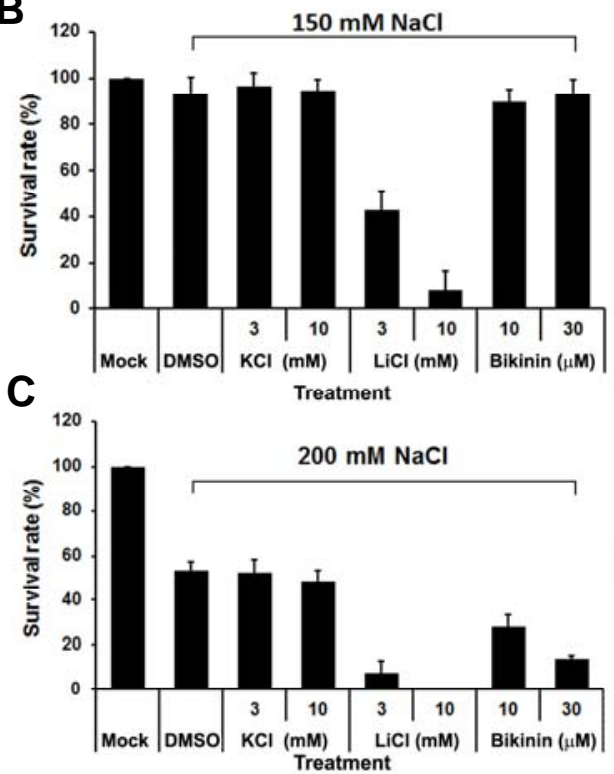

D
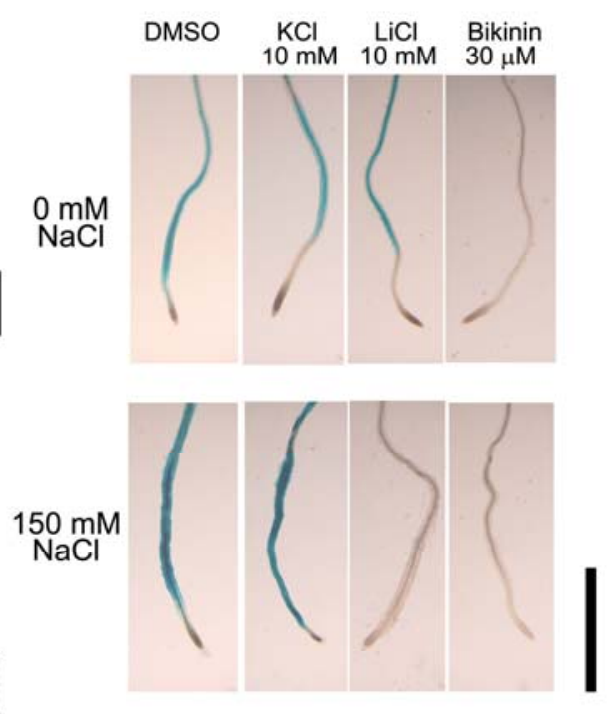

Fig. 4. Reduction in salt stress tolerance upon inhibition of BIN2. (A) Morphology of seedlings treated with mock or various salts. Seedlings were treated separately and arranged in a new plate to obtain a collective image of responses. First row, mock-treated control. Second row, treatment with $\mathrm{KCl}$ or $\mathrm{LiCl}$ alone. Third row, $\mathrm{NaCl}$ treatment in combination with $\mathrm{KCl}$ or $\mathrm{LiCl}$. Scale bar $=5 \mathrm{~mm}$. (B, C) Survival rate of Arabidopsis seedlings grown in $150 \mathrm{mM}$ (B) or $200 \mathrm{mM} \mathrm{NaCl}$ media (C) supplemented with salts or bikinin (a BIN2 inhibitor). Survival rate was measured as the ratio of the number of green vs. pale yellow seedlings. Error bar $=S D(n=3)$. (D) GUS staining pattern in the roots of RD26pro:GUS seedlings. Scale bar $=1 \mathrm{~mm}$.

ed with $3 \mathrm{mM} \mathrm{KCl}$ (Figs. $4 \mathrm{~A}$ and $4 \mathrm{~B}$ ). We obtained the similar results when repeating this experiment using bikinin. Induction of GUS activity in the RD26pro:GUS line by $150 \mathrm{mM} \mathrm{NaCl}$ was also inhibited by $\mathrm{LiCl}$ and bikinin (Fig. 4D and Supplementary Fig. S3), implying that the low survival rate of plants treated with salt and $\mathrm{LiCl}$ was mainly due to the inhibition of ABA-response genes by BRs, especially by the pathway regulated by the BIN2 kinase. Taken together, ABA suppresses the BR pathway, which acts via BIN2, to impart salt stress tolerance.

\section{Conserved motifs in the co-regulated genes}

To further understand how the genes are co-regulated by BRs and $A B A$, we compared the promoter sequences of the gene identified and displayed in Fig. 2. Firstly, we found that the motif sequence conserved among the ABA-upregulated and BRdownregulated genes was clearly an ABRE (motif 1, Fig. 5A). Motif 1 is similar to the BRRE; therefore, it is tempting to suggest that BZR1 and ABF/AREB transcription factors may competitively regulate their target genes by binding to the same response elements.

Secondly, in the case of the BR-up and ABA-downregulated genes, a GAGA motif was enriched (Fig. 5B). Previously, it was reported that BASIC PENTACYSTEINE (BPC) proteins bind to this GAGA motif (Meister et al., 2004; Monfared et al., 2011; Sing et al., 2009). It is likely that BPC may function in the antagonistic regulation of $\mathrm{ABA}$ and $\mathrm{BR}$. However, we cannot rule out the possibility that conserved sequences can be bound by currently unidentified transcription factors (Rozhon et al., 2010; Yan et al., 2009).

\section{DISCUSSION}

BR-deficient mutants are known to be hypersensitive to $A B A$
(Steber and McCourt, 2001; Zhang et al., 2009), suggesting that $A B A$ can efficiently induce $A B A$ responses when growth is minimized. Therefore, we identified the genes antagonistically regulated by $\mathrm{ABA}$ and $\mathrm{BRs}$, including RD26 and the ANAC genes, which were previously shown to function in various stress responses (Christianson et al., 2010). In addition, we provided evidence that BIN2 is necessary to maintain the balance between ABA and BR signaling under salt stress conditions.

\section{Growth inhibition mechanism under stress conditions}

Since it is not fully understood how ABA inhibits growth, we studied the mechanisms controlled by $A B A$ and the growthpromoting BRs. Plants grown in medium supplemented with $\mathrm{ABA}$ or $\mathrm{NaCl}$ displayed a growth retardation phenotype similar to that of BR-deficient mutants (Fig. 1). One explanation for this is that BR downregulates the genes that may not be directly required for stress-tolerance processes. In support of this, the expression of RD26 and other genes encoding stress-related $N A C$ transcription factors was reduced by treatment with epi-BL. Likewise, elongated-D (elg- $D)$, a gain-of-function allele of $B A K 1$ with an enhanced growth phenotype, exhibited reduced photosynthetic efficiency upon salt stress, possibly due to the reduced expression of genes involved in stress responses (Chung et al., 2012). Conversely, a recent microarray analysis revealed that many of the cold stress-responsive genes, including WRKY and CBFs, are upregulated in bri1-9, suggesting that BR signaling antagonistically regulates stress-response genes (Kim et al., 2010).

Salt-treated roots tend to break easily and this effect is severe when $B R$ is added. In contrast, when Pcz is added, roots remain thick and relatively intact compared to those subjected to salt treatment alone (Supplementary Fig. S2). In Arabidopsis, 


Motif 1

Fig. 5. Consensus sequence elements in the promoter of genes co-regulated by both $A B A$ and BRs. The top fourmost over-represented motifs found in the -1000 bp proximal promoter of genes are shown. Genes that are (A) upregulated by ABA and downregulated by BR and (B) downregulated by $A B A$ and upregulated by $B R$. Sequence logos for $(A)$ and $(B)$ were generated using the MEME analysis tool. The total information content of each logo is given in bits; the height of each nucleotide in the logo represents the positional probability of that nucleotide multiplied by the information content of the logo. E-value means the possibility of finding the motifs merely by chance.

cell wall loosening is temporarily required for BR-mediated cell expansion, and this is catalyzed by XYLOGLUCAN ENDOHYDROLASES (XEH) (Fanutti et al., 1993). Previously, it was demonstrated that the salt-responsive transcription factor OSMPS, which downregulates the expression of cell-wall biosynthetic genes and EXPANSIN (EXP) genes, is repressed by growth hormones such as BRs and auxin, suggesting that OsMPS functions in the crosstalk that balances adaptive growth with survival under salt stress (Schmidt et al., 2013). Pcz treatment might have corrected the altered root shape in plants exposed to salt stress by effective disruption of the BR signaling (Supplementary Fig. S2).

It was interesting to observe that $R D 26$ overexpression resulted in semi-dwarfism similar to that observed in BR mutants (Supplementary Fig. S1). This morphological similarity suggests that $R D 26$ antagonistically regulates the expression of growthpromoting genes regulated by BRs. Indeed, transgenic lines overexpressing RD26 or ABF3 and ABF4 consistently displayed a semi-dwarf phenotype, but enhanced drought tolerance (Kang et al., 2002). Taken together, stress-responsive transcription factors appear to limit energy expenditure on growth to enhance stress tolerance, possibly by downregulating BR-responsive genes.

BR and ABA signaling pathways converge at BIN2

Seedlings in which BIN2 activity was inhibited by either $\mathrm{LiCl}$ or bikinin displayed a decreased survival rate when subjected to a salt stress of $200 \mathrm{mM} \mathrm{NaCl}$ (Fig. 4). GUS activity was absent in RD26pro:GUS plants treated with $\mathrm{LiCl}$ and bikinin. These results suggest that BIN2 controls the expression of genes involved in salt stress tolerance, including RD26. BIN2 is a pro- tein kinase that phosphorylates BR-specific transcription factors such as BZR1 and BES1. It is possible that BIN2 regulates stress-associated genes, especially those that are upregulated by $A B A$ and downregulated by $B R$, through controlling the activities of BZR1 and BES1. Additionally, overexpression of AtGSK1, the closest homolog of BIN2, results in enhanced salt stress tolerance in Arabidopsis, suggesting that BIN2 may function in the salt stress response (Piao et al., 2001). Based on these findings, we propose a model that illustrates the interaction between BRs and ABA in controlling growth and stress tolerance (Supplementary Fig. S4).

We predict that salt stress controls a step below BIN2 in the $\mathrm{BR}$ signaling pathway, because $\mathrm{NaCl}$ treatment in combination with BIN2 inhibition altered the expression of RD26 and rendered the seedlings less tolerant than those treated with salt alone. Future research should examine how $\mathrm{NaCl}$ signals modify the activity of BIN2. The underlying mechanism may function directly or indirectly through another signaling component that has yet to be discovered.

In conclusion, we sought to determine why stressed plants display stunted growth. We analyzed the relationship between BRs, which promote growth, and ABA, which is involved in the response to environmental stress. Based on the phenotypic similarities between ABA-treated seedlings and bri1-9, we searched for and identified genes that are oppositely regulated by $A B A$ and $B R s$ in previously reported microarray data sets. Of these genes, RD26, which belongs to the ANAC family, was chosen as representative and shown to be specifically induced by ABA, but suppressed by BRs. We provided evidence that BIN2 plays a key role in balancing the growth and the stress responses during periods of stress. Thus, our results suggest 
that $\mathrm{ABA}$ and $\mathrm{BRs}$ may regulate a set of target genes to finetune Arabidopsis growth during the stress response.

Note: Supplementary information is available on the Molecules and Cells website (www.molcells.org).

\section{ACKNOWLEDGMENTS}

We would like to thank K. Shinozaki for providing the expression constructs and RD26 lines. This research was supported, in part, by grants from the Next-Generation BioGreen 21 Program (Plant Molecular Breeding Center No. PJ008051); the Cooperative Research Program for Agriculture Science \& Technology Development (Project No. PJ906910), funded by the Rural Development Administration; the Technology Development Program (110033-5) for Agriculture and Forestry, Ministry of Agriculture, Food and Rural Affairs, Republic of Korea (to S.C.); and BK21 Research Fellowships (to Y.C.) funded by the Ministry of Education (S.D.G.).

\section{REFERENCES}

Aida, M., Ishida, T., Fukaki, H., Fujisawa, H., and Tasaka, M. (1997). Genes involved in organ separation in Arabidopsis: an analysis of the cup-shaped cotyledon mutant. Plant Cell 9, 841-857.

Bailey, T.L., and Elkan, C. (1995). The value of prior knowledge in discovering motifs with MEM. Proceedings/international conference on intelligent systems for molecular biology. ISMB 3, 2129.

Blazquez, M.A., Green, R., Nilsson, O., Sussman, M.R., and Weigel D. (1998). Gibberellins promote flowering of arabidopsis by activating the LEAFY promoter. Plant Cell 10, 791-800.

Busk, P.K., and Pages, M. (1997). Protein binding to the abscisic acid-responsive element is independent of VIVIPAROUS1 in vivo. Plant Cell 9, 2261-2270.

Choe, S. (2006). Brassinosteroid biosynthesis and inactivation. Physiol. Plant. 126, 539-548.

Choe, S., Fujioka, S., Noguchi, T., Takatsuto, S., Yoshida, S., and Feldmann, K.A. (2001). Overexpression of DWARF4 in the brassinosteroid biosynthetic pathway results in increased vegetative growth and seed yield in Arabidopsis. Plant J. 26, 573-582.

Choe, S., Schmitz, R.J., Fujioka, S., Takatsuto, S., Lee, M.O., Yoshida, S., Feldmann, K.A., and Tax, F.E. (2002). Arabidopsis brassinosteroid-insensitive dwarf12 mutants are semidominant and defective in a glycogen synthase kinase 3-like kinase. Plant Physiol. 130, 1506-1515.

Christianson, J.A., Dennis, E.S., Llewellyn, D.J., and Wilson, I.W. (2010). ATAF NAC transcription factors: regulators of plant stress signaling. Plant Sig. Behav. 5, 428-432.

Chung, Y., and Choe, S. (2013). The Regulation of Brassinosteroid Biosynthesis in Arabidopsis. Crit. Rev. Plant Sci. 32, 396-410.

Chung, Y., Maharjan, P.M., Lee, O., Fujioka, S., Jang, S., Kim, B., Takatsuto, S., Tsujimoto, M., Kim, H., Cho, S., et al. (2011). Auxin stimulates DWARF4 expression and brassinosteroid biosynthesis in Arabidopsis. Plant J. 66, 564-578.

Chung, Y., Choe, V., Fujioka, S., Takatsuto, S., Han, M., Jeon, J.S., Park, Y.I., Lee, K.O., and Choe, S. (2012). Constitutive activation of brassinosteroid signaling in the Arabidopsis elongated-D/bak1 mutant. Plant Mol. Biol. 80, 489-501.

Clouse, S.D., and Sasse, J.M. (1998). BRASSINOSTEROIDS: Essential regulators of plant growth and development. Annu. Rev. Plant Physiol. Plant Mol. Biol. 49, 427-451.

De Rybel, B., Audenaert, D., Vert, G., Rozhon, W., Mayerhofer, J., Peelman, F., Coutuer, S., Denayer, T., Jansen, L., Nguyen, L., et al. (2009). Chemical inhibition of a subset of Arabidopsis thaliana GSK3-like kinases activates brassinosteroid signaling. Chem. Biol. 16, 594-604

Fanutti, C., Gidley, M.J., and Reid, J.S. (1993). Action of a pure xyloglucan endo-transglycosylase (formerly called xyloglucanspecific endo-( $1 \rightarrow 4$ )-beta-D-glucanase). from the cotyledons of germinated nasturtium seeds. Plant J. 3, 691-700.

Fujita, M., Fujita, Y., Maruyama, K., Seki, M., Hiratsu, K., Ohme-
Takagi, M., Tran, L.S., Yamaguchi-Shinozaki, K, and Shinozaki, K. (2004). A dehydration-induced NAC protein, RD26, is involved in a novel ABA-dependent stress-signaling pathway. Plant J. 39, 863-876.

Furihata, T., Maruyama, K., Fujita, Y., Umezawa, T., Yoshida, R., Shinozaki, K., and Yamaguchi-Shinozaki, K. (2006). Abscisic acid-dependent multisite phosphorylation regulates the activity of a transcription activator AREB1. Proc. Natl Acad. Sci. USA 103, 1988-1993.

Grill, E., and Himmelbach, A. (1998). ABA signal transduction. Curr. Opin. Plant Biol. 1, 412-418.

Guiltinan, M.J., Marcotte, W.R., Jr., and Quatrano, R.S. (1990). A plant leucine zipper protein that recognizes an abscisic acid response element. Science 250, 267-271.

Hartwig, T., Corvalan, C., Best, N.B., Budka, J.S., Zhu, J.Y., Choe, S., and Schulz, B. (2012). Propiconazole is a specific and accessible brassinosteroid (BR) biosynthesis inhibitor for Arabidopsis and maize. PLoS One 7, e36625.

He, J.X., Gendron, J.M., Sun, Y., Gampala, S.S., Gendron, N., Sun, C.Q., and Wang, Z.Y. (2005). BZR1 is a transcriptional repressor with dual roles in brassinosteroid homeostasis and growth responses. Science 307, 1634-1638.

Hobo, T., Kowyama, Y., and Hattori, T. (1999). A bZIP factor, TRAB1, interacts with VP1 and mediates abscisic acid-induced transcription. Proc. Natl Acad. Sci. USA 96, 15348-15353.

Jensen, M.K., Lindemose, S., de Masi, F., Reimer, J.J., Nielsen, M., Perera, V., Workman, C.T., Turck, F., Grant, M.R., Mundy, J., et al. (2013). ATAF1 transcription factor directly regulates abscisic acid biosynthetic gene NCED3 in Arabidopsis thaliana. FEBS Open Bio. 3, 321-327.

Jin, H., Yan, Z., Nam, K.H., and Li, J. (2007). Allele-specific suppression of a defective brassinosteroid receptor reveals a physiological role of UGGT in ER quality control. Mol. Cell 26, 821-830.

Kang, J.Y., Choi, H.I., Im, M.Y., and Kim, S.Y. (2002). Arabidopsis basic leucine zipper proteins that mediate stress-responsive abscisic acid signaling. Plant Cell 14, 343-357.

Kim, H.B., Kwon, M., Ryu, H., Fujioka, S., Takatsuto, S., Yoshida, S. An, C.S., Lee, I., Hwang, I., and Choe, S. (2006). The regulation of DWARF4 expression is likely a critical mechanism in maintaining the homeostasis of bioactive brassinosteroids in Arabidopsis. Plant Physiol. 140, 548-557.

Kim, T.W., Guan, S., Sun, Y., Deng, Z., Tang, W., Shang, J.X., Sun, Y., Burlingame, A.L., and Wang, Z.Y. (2009). Brassinosteroid signal transduction from cell-surface receptor kinases to nuclear transcription factors. Nat. Cell Biol. 11, 1254-1260.

Kim, S.Y., Kim, B.H., Lim, C.J., Lim, C.O., and Nam, K.H. (2010). Constitutive activation of stress-inducible genes in a brassinosteroid-insensitive 1 (bri1) mutant results in higher tolerance to cold. Physiol. Plant. 138, 191-204.

Kim, B., Fujioka, S., Kwon, M., Jeon, J., and Choe, S. (2013). Arabidopsis Brassinosteroid-overproducing gulliver3-D/dwarf4-D mutants exhibit altered responses to Jasmonic acid and pathogen. Plant Cell Rep. 32, 1139-1149.

Kim, B., Jeong, Y.J., Corvalan, C., Fujioka, S., Cho, S., Park, T., and Choe, S. (2014). Darkness and gulliver2/phyB mutation decrease the abundance of phosphorylated BZR1 to activate brassinosteroid signaling in Arabidopsis. Plant J. 77, 737-747.

Kinoshita, T., Cano-Delgado, A., Seto, H., Hiranuma, S., Fujioka, S., Yoshida, S., and Chory, J. (2005). Binding of brassinosteroids to the extracellular domain of plant receptor kinase BRI1. Nature 433, 167-171.

Klein, P.S., and Melton, D.A. (1996). A molecular mechanism for the effect of lithium on development. Proc. Natl Acad. Sci. USA 93, 8455-8459.

Lee, S.C., and Luan, S. (2012). ABA signal transduction at the crossroad of biotic and abiotic stress responses. Plant Cell. Environ. 35, 53-60.

$\mathrm{Li}$, J., and Nam, K.H. (2002). Regulation of brassinosteroid signaling by a GSK3/SHAGGY-like kinase. Science 295, 12991301.

Li, J., Nam, K.H., Vafeados, D., and Chory, J. (2001). BIN2, a new brassinosteroid-insensitive locus in Arabidopsis. Plant Physiol. $127,14-22$.

Ma, Y., Szostkiewicz, I., Korte, A., Moes, D., Yang, Y., Christmann, A., and Grill, E. (2009). Regulators of PP2C phosphatase 
activity function as abscisic acid sensors. Science 324, 10641068.

Maharian, P.M., and Choe, S. (2011). High temperature stimulates DWARF4 (DWF4). Expression to increase hypocotyl elongation in Arabidopsis. J. Plant Biol. 54, 425-429.

Meister, R.J., Williams, L.A., Monfared, M.M., Gallagher, T.L., Kraft, E.A., Nelson, C.G., and Gasser, C.S. (2004). Definition and interactions of a positive regulatory element of the Arabidopsis INNER NO OUTER promoter. Plant J. 37, 426-438.

Monfared, M.M., Simon, M.K., Meister, R.J., Roig-Villanova, I., Kooiker, M., Colombo, L., Fletcher, J.C., and Gasser, C.S. (2011). Overlapping and antagonistic activities of BASIC PENTACYSTEINE genes affect a range of developmental processes in Arabidopsis. Plant J. 66, 1020-1031.

Mundy, J., Yamaguchi-Shinozaki, K., and Chua, N.H. (1990). Nuclear proteins bind conserved elements in the abscisic acidresponsive promoter of a rice rab gene. Plant Cell. Environ. 87, 1406-1410.

Nemhauser, J.L., Hong, F., and Chory, J. (2006). Different plant hormones regulate similar processes through largely nonoverlapping transcriptional responses. Cell 126, 467-475.

Noguchi, T., Fujioka, S., Choe, S., Takatsuto, S., Yoshida, S., Yuan, H., Feldmann, K.A., and Tax, F.E. (1999). Brassinosteroidinsensitive dwarf mutants of Arabidopsis accumulate brassinosteroids. Plant Physiol. 121, 743-752.

Ooka, H., Satoh, K., Doi, K., Nagata, T., Otomo, Y., Murakami, K., Matsubara, K., Osato, N., Kawai, J., Carninci, P., et al. (2003). Comprehensive analysis of NAC family genes in Oryza sativa and Arabidopsis thaliana. DNA research: an international journal for rapid publication of reports on genes and genomes 10, 239247.

Park, S.Y., Fung, P., Nishimura, N., Jensen, D.R., Fujii, H., Zhao, Y., Lumba, S., Santiago, J., Rodrigues, A., Chow, T.F., et al. (2009). Abscisic acid inhibits type $2 \mathrm{C}$ protein phosphatases via the PYR/PYL family of START proteins. Science 324, 1068-1071.

Peng, P., Yan, Z., Zhu, Y., and Li, J. (2008). Regulation of the Arabidopsis GSK3-like kinase BRASSINOSTEROID-INSENSITIVE 2 through proteasome-mediated protein degradation. Mol. Plant 1 , 338-346.

Piao, H.L., Lim, J.H., Kim, S.J., Cheong, G.W., and Hwang, I. (2001) Constitutive over-expression of AtGSK1 induces $\mathrm{NaCl}$ stress responses in the absence of $\mathrm{NaCl}$ stress and results in enhanced $\mathrm{NaCl}$ tolerance in Arabidopsis. Plant J. 27, 305-314.

Rozhon, W., Mayerhofer, J., Petutschnig, E., Fujioka, S., and Jonak, C. (2010). ASKtheta, a group-III Arabidopsis GSK3, functions in the brassinosteroid signalling pathway. Plant J. 62, 215-223.

Russinova, E., Borst, J.W., Kwaaitaal, M., Cano-Delgado, A., Yin, Y., Chory, J., and de Vries, S.C. (2004). Heterodimerization and endocytosis of Arabidopsis brassinosteroid receptors BRI1 and AtSERK3 (BAK1).. Plant Cell 16, 3216-3229.

Sakuma, Y., Maruyama, K., Osakabe, Y., Qin, F., Seki, M., Shinozaki, K., and Yamaguchi-Shinozaki, K. (2006). Functional analysis of an Arabidopsis transcription factor, DREB2A, involved in drought-responsive gene expression. Plant Cell 18, 1292-1309.

Schmidt, R., Schippers, J.H., Mieulet, D., Obata, T., Fernie, A.R., Guiderdoni, E., and Mueller-Roeber, B. (2013). MULTIPASS, a rice R2R3-type MYB transcription factor, regulates adaptive growth by integrating multiple hormonal pathways. Plant J. 76 258-273.

Shinozaki, K., and Yamaguchi-Shinozaki, K. (2007). Gene networks involved in drought stress response and tolerance. J. Exp. Bot. $58,221-227$

Sing, A., Pannell, D., Karaiskakis, A., Sturgeon, K., Djabali, M., Ellis, J., Lipshitz, H.D., and Cordes, S.P. (2009). A vertebrate Polycomb response element governs segmentation of the posterior hindbrain. Cell 138, 885-897.

Stambolic, V., Ruel, L., and Woodgett, J.R. (1996). Lithium inhibits glycogen synthase kinase- 3 activity and mimics wingless signalling in intact cells. Curr. Biol. 6, 1664-1668.

Steber, C.M., and McCourt, P. (2001). A role for brassinosteroids in germination in Arabidopsis. Plant Physiol. 125, 763-769.

Sun, Y., Fan, X.Y., Cao, D.M., Tang, W., He, K., Zhu, J.Y., He, J.X., Bai, M.Y., Zhu, S., Oh, E., et al. (2010). Integration of brassinosteroid signal transduction with the transcription network for plant growth regulation in Arabidopsis. Dev. Cell 19, 765-777.

Sun, Y., Han, Z., Tang, J., Hu, Z., Chai, C., Zhou, B., and Chai, J. (2013). Structure reveals that BAK1 as a co-receptor recognizes the BRI1-bound brassinolide. Cell Res. 23, 1326-1329.

Tang, W., Kim, T.W., Oses-Prieto, J.A., Sun, Y., Deng, Z., Zhu, S. Wang, R., Burlingame, A.L., and Wang, Z.Y. (2008). BSKs mediate signal transduction from the receptor kinase BRI1 in Arabidopsis. Science 321, 557-560

Tran, L.S., Nakashima, K., Sakuma, Y., Simpson, S.D., Fujita, Y., Maruyama, K., Fujita, M., Seki, M., Shinozaki, K., and Yamaguchi-Shinozaki, K. (2004). Isolation and functional analysis of Arabidopsis stress-inducible NAC transcription factors that bind to a drought-responsive cis-element in the early responsive to dehydration stress 1 promoter. Plant Cell 16 2481-2498

Uno, Y., Furihata, T., Abe, H., Yoshida, R., Shinozaki, K., and Yamaguchi-Shinozaki, K. (2000). Arabidopsis basic leucine zipper transcription factors involved in an abscisic aciddependent signal transduction pathway under drought and highsalinity conditions. Proc. Natl Acad. Sci. USA 97, 11632-11637.

Vriet, C., Russinova, E., and Reuzeau, C. (2013). From squalene to brassinolide: the steroid metabolic and signaling pathways across the plant kingdom. Mol. Plant 6, 1738-1757.

Wang, X., and Chory, J. (2006). Brassinosteroids regulate dissociation of $\mathrm{BKI} 1$, a negative regulator of $\mathrm{BR} 11$ signaling, from the plasma membrane. Science 313, 1118-1122.

Wang, X., Kota, U., He, K., Blackburn, K., Li, J., Goshe, M.B., Huber, S.C., and Clouse, S.D. (2008). Sequential transphosphorylation of the BRI1/BAK1 receptor kinase complex impacts early events in brassinosteroid signaling. Dev. Cell 15, 220-235.

Yamaguchi-Shinozaki, K., Mundy, J., and Chua, N.H. (1990). Four tightly linked rab genes are differentially expressed in rice. Plant Mol. Biol. 14, 29-39.

Yan, Z., Zhao, J., Peng, P., Chihara, R.K., and Li, J. (2009). BIN2 functions redundantly with other Arabidopsis GSK3-like kinases to regulate brassinosteroid signaling. Plant Physiol. 150, 710721.

Zhang, S., Cai, Z., and Wang, X. (2009). The primary signaling outputs of brassinosteroids are regulated by abscisic acid signaling. Proc. Natl Acad. Sci. USA 106, 4543-4548. 